Using Fuzzy Set Theory to Assess Country-of-Origin Effects on the Formation of Product Attitude.

Peer-reviewed author version

BRIJS, Kris; VANHOOF, Koen; BRIJS, Tom \& KARLIS, Dimitris (2006) Using Fuzzy

Set Theory to Assess Country-of-Origin Effects on the Formation of Product

Attitude.. In: Torra, V. \& Narukawa, Y. \& Valls, A. \& Domingo-Ferrer, J. (Ed.)

Modeling Decisions for Artificial Intelligence, 3th International Conference. p. 138-149..

Handle: http://hdl.handle.net/1942/1488 


\title{
Using Fuzzy Set Theory to Assess Country-of-Origin Effects on the Formation of Product Attitude
}

\author{
Kris Brijs ${ }^{1}$, Koen Vanhoof ${ }^{1}$, Tom Brijs ${ }^{1}$, and Dimitris Karlis ${ }^{2}$ \\ ${ }^{1}$ Hasselt University, Department of Applied Economics, Agoralaan, gebouw D, \\ 3590 Diepenbeek, Belgium \\ ${ }^{2}$ Athens University of Economics and Business, Department of Statistics, \\ 76 Patision Street, 10434 Athens, Greece \\ \{kris.brijs, koen.vanhoof, tom.brijs\}@uhasselt.be, \\ karlis@aueb.gr
}

\begin{abstract}
Several researchers on country-of-origin (coo) have expressed their interest in knowing how consumers' emotional reactions toward coo-cues affect product attitude formation. This paper shows how Fuzzy Set Theory might serve as a useful approach to that problem. Data was gathered by means of selfadministered questionnaires. Technically, orness of OWA-operators enabled us to distinguish consumers expressing highly positive versus less positive emotions toward coo. It appeared that this variance in emotional estate goes together with a difference in aggregating product-attribute beliefs.
\end{abstract}

\section{Introduction to Product Attitude Formation}

We start this paper by providing a short overview of the literature on product attitude formation. Without going into all the details, it provides the larger (marketing) context in which the technical contribution of our paper should be seen. The motivation for this is that the contribution of this paper does not only lay in technical aspects of the OWA operator (see section 5), but it also provides superior consumer information with managerial relevance that can not be offered by conventional statistical techniques that have been used for this kind of marketing research.

Attitude Theory states that consumers' behaviour toward products is determined to a large extent by their attitude toward them. In line with Peter et al. [19], we define 'attitude' as a person's overall evaluation of a concept. According to Eagly and Chaiken [6], this overall evaluative judgement can be seen as a psychological tendency that expresses some degree of (dis)favour toward the attitude object. The Expectancy Value Model developed by Fishbein and Ajzen [10] posits that this overall evaluative judgement of the product is mediated by the evaluation of salient beliefs. In other words, people combine or integrate product knowledge to form an overall evaluation of products. Thus, consumers' beliefs about product attributes are considered as crucial determinants of their attitude toward the product. The literature on advertising and emotions has challenged some of the basic principles behind this socalled multi-attribute theory. Its key-proposition was that advertisements can generate several affective reactions which also influence the formation of consumers' attitudes toward products. Peterson et al. [20] for instance, stated that ad evoked affects can 
play various roles in consumer decision making, ranging from influencing the ways in which information is processed and stored in memory to determining product choices.

Past research indicates that advertising cues indeed produce different types of affective reactions. According to Derbaix and Pham [5], these range from emotions, feelings, moods and temperaments to preference, attitude and appreciation. As recommended by Verlegh [26], our attention more specifically goes to 'feelings'.

As put by Burke and Edell [2], ad evoked feelings influence consumers' brand attitude through their attitude toward the ad or via their brand attribute beliefs. Holbrook and Batra [13] also found that ad attitude mediates the effect of ad feelings on brand attitude. In addition, they tested whether ad evoked feelings had a direct impact on brand attitude and found that it was rather limited. Results reported by Stayman and Aaker [22] went in the same direction, although they established that the effects of ad feelings on brand attitude were not always necessarily mediated by ad attitude. Yet, on the average, we can state that ad evoked feelings rather exert an indirect influence on the consumer's attitude toward the product. Ad attitude and product attribute beliefs both seem to function as important mediators. Since the concept of ad attitude is not within our scope of interest, we will further concentrate on the other path where feelings evoked by advertising cues like country-of-origin are posited to influence the consumer's overall evaluative judgement of the product indirectly, that is, via the formation and subsequent processing of attribute beliefs.

\section{Coo-Effects: The Affective Approach}

Although coo-effects have been traditionally approached from an information theoretic perspective, several scholars working within the field already argued attention should be paid to the coo-cue's capacity to evoke all kinds of symbolic and emotional connotations which might interfere with the consumer's intent to evaluate a product. Some interesting examples in support of this assumption have been cited by Obermiller and Spangenberg [17]. For instance, they mention the negative reaction toward high quality Israeli-made precision instruments expressed by Americans of Arab origin. Friedman [11] in turn, explains the American Jews' boycott of German-made products during the first decades after the Second World War by the fact that 'Made in Germany' labels elicited all kinds of traumatic feelings. Klein et al. [15] established that previous or ongoing military, political and economic events between Japan and the People's Republic of China generate feelings of 'animosity' affecting Chinese consumers' buying decisions. Still recently, Verlegh [26] demonstrated how Dutch consumers' tendency to identify with their home country is accompanied by less positive feelings toward a foreign coo. These in turn, appeared to influence the formation of beliefs about the attributes of products coming from abroad in a negative way.

Thus, we might conclude that the emotional reactions triggered by the coo-cue act as potential determinants of the consumer's attitude toward foreign sourced products. However, it still remains unclear how they affect this process of product attitude formation. Our attention will be focused on that problem. Throughout the following sections, we will first elaborate on our conception of attitudes. In our effort to explain how coo-related emotions might affect the consumer's product attitude, we will base ourselves on insights from the literature on ad emotions. 


\section{Coo Emotions and Information Processing}

According to Isen [14], affective reactions evoked by ads influence our cognitive activities in many ways. As she puts it, "[...] the evidence suggests that rather than causing people not to think, affect (at least some affects) can influence thought by influencing what people think about, how they relate things to one another, what they try to accomplish, and how they go about solving problems. Thus feelings can have a substantial influence on thought processes and resultant behaviour." [14]. Before turning to our vision on the functioning of emotions evoked by advertising cues like the product's coo, we will briefly review some insights coming from the literature on coo-emotions. To begin with, it is striking to establish how the majority of these studies are concentrated on the functioning of rather extreme negative feelings like animosity [15], ethnocentrism [21], or patriotism [12]. Overall, it is found that these are directly transferred to the product. This leads to situations where people decide not to buy, purely based on their aversive feelings toward the product's coo.

Verlegh [26] wondered whether such powerful effects would also be triggered by milder affective reactions toward coo. As he argued, extremely negative feelings toward foreign nations only manifest themselves in very particular occasions and cannot always be generalized to the context of daily life. In his opinion, the average consumer will rather be characterized by the expression of less intensive feelings toward other countries. Therefore, he focused more on the role of softer feelings. More in detail, he proposed a framework where such weaker coo-related feelings are modelled as determinants of the consumer's product attribute beliefs. Although partial and inconsistent, he found significant supportive evidence in both cases of positive and negative feelings toward coo. Thus, it appears that milder coo-feelings bias our perception of a product's attributes.

However, Han [12] thinks people's perception of a product's quality attributes is not fundamentally determined by the way we feel about the place where it was made. Obermiller and Spangenberg [17] subscribe to this reasoning in positing that consumers who experience extremely negative feelings toward certain countries still acknowledge that products from those nations are of superior quality. Thus, coo-specific feelings apparently do not alter our perception of a product's quality attributes per se, even if we vividly experience them. Han [12] designed a study to examine this problem and found that consumers expressing positive feelings toward the product' $\mathrm{s}$ coo only tentatively rated that product's attributes more favourably.

Thus, in general, it seems that for weaker feelings toward coo, some doubts remain on how they precisely affect our product attitudes. Verlegh [26] thinks they determine our perception of a product's quality attributes albeit that his results and those obtained by others are not very consistent. The question of knowing how such milder affective reactions toward coo influence product attitude formation thus remains open.

Our key proposition will be that softer coo-specific feelings will influence the way in which consumers process these attribute beliefs. More in detail, we argue that less intensive coo-specific feelings will affect the way in which consumers cognitively combine or integrate their product attribute beliefs.

Our reasoning is based on the principles behind the encoding-specificity mechanism developed by Tulving and Thomson [25]. The underlying idea is that affects experienced by individuals can activate thoughts which have been stored in memory as relevant and related to those affects. As put by Cacioppo and Petty, affects indeed 
can "bias issue-relevant thinking by making affectively consonant thoughts and ideas more accessible in memory." [3]. Isen [14] continues that several studies have shown how people being happy show better recall of positive material. Thus, it appears that affective reactions elicited by ads can lead to greater receptiveness of positive or persuasive communication. In line with this reasoning, we assume that individuals will be more inclined to process those particular attribute beliefs which correspond best with their actual emotional state. Therefore, we formulate the following hypothesis:

H: Consumers expressing more positive feelings toward the product's country-of-origin will process the stronger valued attribute beliefs while consumers expressing less positive feelings toward the product's country-of-origin will process the weaker valued attribute beliefs.

\section{Methodology}

A study was designed to determine how feelings evoked by coo-cues influence the respondents' cognitive attribute processing. More specifically, the products selected for our study were DVD-players (utilitarian) and beer (hedonic). The decision to opt for two distinct types of products was taken in order to increment the external validity of our study. Additional motivation for the selection of these two products can be found in the frequent use that is made of them by other coo-researchers. The countries-of-origin selected for our study were Spain and Denmark. Both countries were sufficiently familiar to our respondents and mutually different on a number of country-specific aspects. This made participants feel confident enough in filling out the questionnaire. Also, we obtained two samples of which the overall level or intensity of country-specific feelings aroused substantially varied.

As evaluation function we have chosen the ordered averaging operator (OWA). This operator was originally introduced by Yager [29] to provide a means for aggregating scores with the satisfaction of multiple criteria, which unifies in one operator both conjunctive and disjunctive behaviour. Examples of alternative aggregation operators include the Weighted Mean and the Weighted OWA [23]. However, we have chosen the OWA because the orness-measure can be directly learned from the data.

More formally, an OWA operator [30] of dimension $n$ is a mapping :

$$
f: R^{n} \rightarrow R
$$

that has an associated weighting vector $W$

$$
W=\left[\begin{array}{llll}
W_{1} & W_{2} & \ldots & W_{n}
\end{array}\right]^{T}
$$

such that

$$
\sum_{i} W_{i}=1 \quad, \quad W_{i} \in[0,1]
$$

and where

$$
f\left(a_{1}, \ldots, a_{n}\right)=\sum_{j=1}^{n} W_{j} b_{j}
$$


where $b_{j}$ is the $j$-th largest element of the collection of the aggregated objects $a_{l}, a_{2}$, $\ldots, a_{n}$. The function value $f\left(a_{1}, \ldots, a_{n}\right)$ determines the aggregated value of arguments $a_{1}, a_{2}, \ldots, a_{n}$.

A fundamental aspect of the OWA operator is the re-ordering step, in particular an argument $a_{l}$ is not associated with a particular weight $w_{i}$ but rather a weight $w_{i}$ is associated with a particular ordered position $i$ of the arguments. A known property of the OWA operators is that they include the Max, Min and arithmetic mean operators for the appropriate selection of the vector $W$.

The operator has proven to be very useful because of its versatility and its measure that can quantify or express the nature of the behaviour of the evaluator like pessimistic or optimistic. This measure, called the 'orness measure' of the aggregation, is defined as

$$
\operatorname{orness}(W)=\frac{1}{n-1} \sum_{i=1}^{n}(n-i) W_{i}
$$

As suggested by Yager [30] this measure, which lies in the unit interval, characterizes the degree to which the aggregation is like an or (Max) operation. It can be shown that:

$$
\begin{aligned}
& \operatorname{orness}\left(\left[\begin{array}{llll}
1 & 0 & \ldots & 0
\end{array}\right]^{\mathrm{T}}\right)=1, \\
& \text { orness }\left(\left[\begin{array}{llll}
0 & 0 & \ldots & 1
\end{array}\right]^{\mathrm{T}}\right)=0, \\
& \text { orness }\left(\left[\begin{array}{llll}
1 / n & 1 / n & \ldots & 1 / n
\end{array}\right]^{\mathrm{T}}\right)=0.5
\end{aligned}
$$

Therefore the Max, Min and arithmetic mean operators can be regarded as OWA operators with degree of orness, respectively, 1,0 and 0.5 . The orness measure can be seen as the optimistic degree of the evaluator. The interested reader can find more information on the orness of an aggregation in [8].

Data was gathered by means of two surveys (one for Spain/Spanish products and one for Denmark/Danish products). These were distributed to respectively 616 and 609 graduate students of Belgian nationality. The questionnaire was always administered at the beginning of a regular classroom session. The use of student samples for studying coo-effects is encouraged by Baughn and Yaprak [1] because of their homogeneous composition. In addition, several meta-analyses [16, 27] report that there are no significant differences in the estimates of coo-effects sizes between student and non-student samples. The questionnaire consisted of 4 sections. First, subjects indicated sex and age. The second section included a multi-item measure of subjects' feelings toward coo. The PANAS scale [28] served as a basis for operationalization. More in detail, it consists of 20 items that describe different emotional states. We limited ourselves to the 10 items referring to positive emotions. For each of these, 
subjects had to indicate on a 7-point semantic differential scale how intensively they felt the item in question. The decision to limit ourselves to the use of items standing for positive feelings is based on our motivation to concentrate explicitly on the role of milder positive feelings toward coo. However, as will be pointed out later, after filling out the questionnaire, both samples were subdivided into a group of subjects expressing high positive feelings (137 cases for Spain vs. 194 cases for Denmark) toward coo and into another group of individuals showing less positive coo-specific feelings (134 cases for Spain vs. 74 cases for Denmark), based on the emotion scores.

The third section contained two 4-item scales measuring subjects' beliefs about DVD-player- and beer attributes. For each item, respondents had to indicate on a 7-point Likert scale ranging from 1 [definitely not agree] to 7 [fully agree] whether they believed the product to possess the attribute in question. For both products, the items (i.e., reliability, durability, performance and easiness of use for DVD-players and taste, naturalness, aroma and prestige for beer) were extracted from the cooliterature. Finally, subjects' evaluative judgement of DVD-players and beer was measured by means of a single-item 7-point semantic differential scale probing for the quality of the product.

Given are a collection of $m$ respondents (observations) each comprised of an $n$-tuple of belief values $\left(a_{k 1}, a_{k 2}, \ldots, a_{k n}\right)$ called the arguments (i.e., reliability, durability, performance and easiness of use for DVD-players and taste, naturalness, aroma and prestige for beer), and an associated single value called the aggregated value (i.e., the quality of the product), which we shall denote as $d_{k}$.

Our goal will be to obtain an OWA operator, a weighting vector $W$ that models the process of aggregation and its associated orness measure. We need a OWA operator, $W$, such that for a given group of respondents the following condition is satisfied as much as possible for any $k$ :

$$
f\left(a_{k 1}, a_{k 2}, \ldots, a_{k n}\right)=d_{k}
$$

We shall relax this formulation by looking for a vector of OWA weights $W=$ $\left[\begin{array}{llll}w_{1} & w_{2} & \ldots & w_{n}\end{array}\right]^{\mathrm{T}}$ that approximates the aggregation operator by minimizing the instantaneous errors $e_{k}$ where

$$
e_{k}=\frac{1}{2}\left(b_{k 1} w_{1}+b_{k 2} w_{2}+\ldots+b_{k n} w_{n}-d_{k}\right)^{2}
$$

The situation is complicated by the fact that the above minimization problem is a constrained optimization problem, since the OWA weights $w_{i}$ have to satisfy the following two properties:

$$
\begin{aligned}
& \sum_{i=1}^{n} w_{i}=1 ; \\
& w_{i} \in[0,1], \quad i=(1, \ldots, n) .
\end{aligned}
$$


Therefore, the following transformation is introduced:

$$
W_{i}=\frac{e^{\lambda_{i}}}{\sum_{j=1}^{n} e^{\lambda_{j}}}
$$

From the above transformation it becomes clear that for any values of the parameters $\lambda_{i}$ the weights $w_{i}$ will be positive and will sum to 1 . Therefore, the constrained minimization problem is transformed to the following unconstrained nonlinear programming problem:

Minimize the instantaneous errors $e_{k}$ :

$$
e_{k}=\frac{1}{2}\left(b_{k 1} \frac{e^{\lambda_{1}}}{\sum_{j=1}^{n} e^{\lambda_{j}}}+b_{k 2} \frac{e^{\lambda_{2}}}{\sum_{j=1}^{n} e^{\lambda_{j}}}+\ldots+b_{k n} \frac{e^{\lambda_{n}}}{\sum_{j=1}^{n} e^{\lambda_{n}}}-d_{k}\right)^{2}
$$

with respect to the parameters $\lambda_{i}$.

The gradient descent method was used to learn the weights [9]. See Torra [24] for an alternative estimation method.

\section{Statistical Inference}

The methodology described above measures the orness from a sample rather than a population and hence it is susceptible to random error. It would be interesting to infer statistically about the results based on a sample. Such inference could answer questions whether the orness (or any other similar measure) differs between different groups, to construct confidence intervals for the quantities under investigation and to test hypotheses for the population values. To our knowledge, however, there is no such technique for statistical inference available. Derivation of theoretical results is not simple because of the complicated nature of the measurements. For this reason, we base our statistical inference on resampling methods, namely non-parametric bootstrap. We construct confidence intervals for the orness measure based on nonparametric bootstrap. Bootstrap is a recently fashionable way for statistical inference for quantities for which theoretical and/or even asymptotic results are hard to derive. In these cases simulated inference based on bootstrap [7] is a key tool for inference. Each resample is analyzed exactly as if it were for the real data. To implement the non-parametric bootstrap, observations are sampled with replacement from the original data set until sample size is equal to that for the real data. These observations comprise the first bootstrap resample, denoted as $X_{1}^{*}$. The process is repeated a number of B times, and we end up with B resamples, denoted by $X_{1}^{*}, X_{2}^{*}, \ldots, X_{B}^{*}$. The key idea is that all these resamples can be considered as samples from the unknown population (or at least they look like the unknown population).

Now, denote the orness measure based on sample $X_{i}^{*}$ as $O_{i}^{*}$. Hence if we calculate the orness (or any other measure) for all the $\mathrm{B}$ resamples, we have $\mathrm{B}$ realizations 
of the quantity of interest $O_{1}^{*}, O_{2}^{*}, \ldots, O_{B}^{*}$, and in fact we have a random sample from the sampling distribution of this quantity. Hence, as the sample mean estimates the unknown population mean, we can estimate every quantity of interest based on those $B$ values. By this approach, we can estimate variances, biases or any other quantity of interest including the construction of confidence intervals. The standard deviation for the orness will be simply the standard deviation of the values $O_{1}^{*}, O_{2}^{*}, \ldots, O_{B}^{*}$, i.e.

$$
s(\hat{O})=\sqrt{\frac{1}{B-1} \sum_{i=1}^{B}\left(O_{i}^{*}-\bar{O}\right)^{2}}
$$

where $\bar{O}=\frac{1}{B} \sum_{i=1}^{B} O_{i}^{*}$.

There are several different ways to construct confidence intervals based on bootstrap values. We adapt the simple quantile based confidence intervals and hence a $95 \%$ confidence interval is constructed as $\left[k_{0.025}, k_{0.975}\right]$,where $k_{a}$ is the a\% sample quantile of the bootstrap values $O_{1}^{*}, O_{2}^{*}, \ldots, O_{B}^{*}$.

In a similar fashion, one can construct confidence intervals for any quantity of interest as for example for the $w_{i}$ 's. We emphasize that for the latter the standard approach to treat them as merely proportions is not correct as they are correlated proportions since they have to sum to one. Our bootstrap approach creates correct intervals in the sense that it takes into account the correlation structure that exists.

\section{Results}

Table 1-4 below present the results of the orness measure and the OWA weights (with standard errors between brackets) for Spanish/Danish DVD players and beer based on the outcome of the questionnaire. More specifically, these tables show the results for three groups of respondents. The first group is always the entire sample (616 cases for Spanish survey vs. 609 cases for Danish survey), whilst the second and third group are those respondents expressing respectively high positive feelings toward coo (137 cases for Spain vs. 194 cases for Denmark) and rather low positive feelings toward coo (134 cases for Spain vs. 74 cases for Denmark). Standard errors are based on $\mathrm{B}=1000$ bootstrap replications using the procedure described above.

When comparing the group of respondents with high positive feelings toward coo (say group A) versus those expressing less positive feelings toward coo (say group B), table 1 (i.e., results for Spanish DVD-players) shows that the orness measure for group A is higher than for group B. When constructing $95 \%$ confidence intervals we found that for group A the interval is $(0.439,0.672)$, while for group B $(0.347,0.521)$, which implies a certain overlap. Statistically speaking, the differences between group $\mathrm{A}$ and $\mathrm{B}$ are not significant on a 5\% level. According to our bootstrap results, it is however significant on the $10 \%$ although this decision depends on the bootstrap experiment used. Qualitatively, however, it is clear that group A has a larger orness, which somehow confirms our hypothesis that people expressing high positive feelings 
Table 1. Results for Spanish DVD players

\begin{tabular}{l|ccccc}
\hline Data set & Orness & $w_{1}$ & $w_{2}$ & $w_{3}$ & $w_{4}$ \\
\hline All cases (616) & 0.4742 & 0.1338 & 0.2758 & 0.4695 & 0.1207 \\
& $(0.0259)$ & $(0.0326)$ & $(0.0858)$ & $(0.0870)$ & $(0.0382)$ \\
Group A: (137) & 0.5499 & 0.1931 & 0.3866 & 0.2971 & 0.1230 \\
& $(0.0619)$ & $(0.0682)$ & $(0.2188)$ & $(0.2191)$ & $(0.0734)$ \\
Group B: (134) & 0.4061 & 0.1495 & 0.2065 & 0.3567 & 0.2871 \\
& $(0.0556)$ & $(0.0560)$ & $(0.1350)$ & $(0.1734)$ & $(0.1124)$ \\
Significance & $S$ & $N S$ & $N S$ & $N S$ & $N S$ \\
\hline
\end{tabular}

Table 2. Results for Spanish beer

\begin{tabular}{l|ccccc}
\hline Data set & Orness & $w_{1}$ & $w_{2}$ & $w_{3}$ & $w_{4}$ \\
\hline All cases (616) & 0.4290 & 0.1554 & 0.3171 & 0.1866 & 0.3407 \\
& $(0.0220)$ & $(0.0299)$ & $(0.0706)$ & $(0.0798)$ & $(0.0471)$ \\
Group A: (137) & 0.4489 & 0.2015 & 0.1483 & 0.4452 & 0.2047 \\
& $(0.0521)$ & $(0.0620)$ & $(0.1568)$ & $(0.1983)$ & $(0.1038)$ \\
Group B: (134) & 0.3438 & 0.1824 & 0.1907 & 0.1023 & 0.5243 \\
& $(0.0443)$ & $(0.0666)$ & $(0.1070)$ & $(0.1217)$ & $(0.0938)$ \\
Significance & $N S$ & $N S$ & $N S$ & $S$ & $S$ \\
\hline
\end{tabular}

Table 3. Results for Danish DVD players

\begin{tabular}{l|ccccc}
\hline Data set & Orness & $w_{1}$ & $w_{2}$ & $w_{3}$ & $w_{4}$ \\
\hline All cases (609) & 0.5265 & 0.1901 & 0.3774 & 0.2544 & 0.1780 \\
& $(0.0215)$ & $(0.0473)$ & $(0.07370$ & $(0.0651)$ & $(0.0310)$ \\
Group A: (194) & 0.5336 & 0.2491 & 0.2712 & 0.3107 & 0.1688 \\
& $(0.0504)$ & $(0.0919)$ & $(0.1652)$ & $(0.1670)$ & $(0.0595)$ \\
Group B: (74) & 0.5133 & 0.1727 & 0.3005 & 0.4207 & 0.1060 \\
& $(0.0582)$ & $(0.1030)$ & $(0.1934)$ & $(0.1867)$ & $(0.0902)$ \\
Significance & $N S$ & $N S$ & $N S$ & $N S$ & $N S$ \\
\hline
\end{tabular}

Table 4. Results for Danish beer

\begin{tabular}{l|ccccc}
\hline Data set & Orness & $w_{1}$ & $w_{2}$ & $w_{3}$ & $w_{4}$ \\
\hline All cases (609) & 0.4216 & 0.2099 & 0.1683 & 0.2983 & 0.3233 \\
& $(0.0204)$ & $(0.0372)$ & $(0.0732)$ & $(0.0759)$ & $(0.0399)$ \\
Group A: (194) & 0.4166 & 0.2399 & 0.1223 & 0.2855 & 0.3522 \\
& $(0.0357)$ & $(0.0699)$ & $(0.1116)$ & $(0.1205)$ & $(0.0780)$ \\
Group B: (74) & 0.3733 & 0.2266 & 0.0775 & 0.2849 & 0.4109 \\
& $(0.0548)$ & $(0.07800$ & $(0.1238)$ & $(0.1406)$ & $(0.0867)$ \\
Significance & $N S$ & $N S$ & $N S$ & $N S$ & $N S$ \\
\hline
\end{tabular}

toward coo tend to use a more optimistic evaluation function toward evaluating the quality of Spanish DVD-players. In other words, they tend to base their quality evaluation more on the more positively evaluated attributes. 
Confirmation of the encoding-specificity principle should, however, also be reflected by the individual OWA weights $\left(w_{1}\right.$ to $\left.w_{4}\right)$ such that for group A versus group $\mathrm{B}$, the ordered weights $w_{1}$ and $w_{2}$ should show higher values and the ordered weights $w_{3}$ and $w_{4}$ should show lower values. Based on results depicted in table 1 it can be observed that indeed $w_{1}$ and $w_{2}$ are higher in group A compared to group B. However, their individual differences are not statistically significant. Similarly, it can be seen from the values for $w_{3}$ and $w_{4}$ that they are higher in group B compared to group A, although their individual differences are again not statistically significant.

Table 2 presents the results obtained for Spanish beer. Also in this case, the orness measure for group A is surpassing that for group B, although in this case the difference is not statistically significant. The $95 \%$ confidence interval for group A is $(0.351,0.555)$ while for group B $(0.262,0.435)$. Yet, there is a clear indication that group A has a larger orness. This can again be seen as supportive evidence for our hypothesis. Thus, one could conclude that respondents with high positive coo-feelings tend to base their quality evaluation of Spanish beer rather on the more favourably evaluated attributes. However, in this case the results for the weight values are less convincing since the value of $w 2$ is larger in group B than in group A, and the value of $w_{3}$ is larger in group A than in group B.

Table 3 and 4 show the results for Danish DVD-players and beer. Although there is a tendency that the orness is again slightly higher for group A than for group B, the differences are much smaller compared to the results for Spain and not statistically significant. For example, for Danish DVD-players, the 95\% confidence interval for group $\mathrm{A}$ is $(0.435,0.626)$ and for group $\mathrm{B}(0.402,0.641)$, showing a large overlap. With respect to the values of $w_{1}$ to $w_{4}$ the results are not consistent.

Overall, it is interesting to observe that we can find much more evidence for our hypothesis in the case of Spanish products compared to Danish products.

\section{Conclusion}

From a practical point of view, our study shows how milder coo-specific feelings serve as a useful device for advertisers to direct consumers' processing of attribute beliefs. More in detail, their functioning can be understood as some kind of encodingspecificity mechanism. That is, consumers during their product evaluation ascribe most importance to those attribute beliefs which are closer in line with their internal affective state. Interestingly, support for our hypothesis was somewhat more substantial for the Spanish than for the Danish survey. Thus, the type of country seems to play a role in determining to what extent the encoding-specificity mechanism manifests itself.

From a technical point of view, we opted for an alternative methodology in using the OWA-operator. In our opinion, this is a useful approach while the interpretation of the OWA-weights is more straightforward compared to the more complex LISREL-models as they have been traditionally used for instance by Han [12]. An additional advantage lies in the fact that the 'orness measure' gives us the needed quantification of the optimistic degree of an evaluation. This aspect alone is already a huge advantage of the fuzzy set approach compared to the more traditional LISREL approaches where this degree of optimism cannot be extracted from the data. Finally, 
we introduced a bootstrap procedure to estimate the orness and the level of uncertainty around it. This enables us to construct confidence intervals and conduct hypothesis tests. As far as we know, estimating this degree of uncertainty of the orness measure has never been introduced in the literature before.

\section{References}

1. Baughn, C.C., Yaprak, A.: Mapping Country-of-Origin Research: Recent Developments and Emerging Avenues. In: Papadopoulos, N., Heslop, L.A. (eds.): Product-Country Images: Importance and Role in International Marketing. International Business Press, New York (1993)

2. Burke, M.C., Edell, J.A.:The Impact of Feelings on Ad-Based Affect and Cognition. Journal of Marketing Research, 26 (February) (1989) 69-83

3. Cacioppo, J.T., Petty, R.E.: The Elaboration Likelihood Model: The Role of Affect and Affect-Laden Information Processing in Persuasion. In: Cafferata, P., Tybout, A.M. (eds.): Cognitive and Affective Responses to Advertising. Lexington Books, Toronto (1989)

4. Cafferata, P., Tybout, A.M.: Cognitive and Affective Responses to Advertising. Lexington Books, Toronto (1989)

5. Derbaix, C., Pham, M.T.: For the development of measures of emotion in marketing: Summary of prerequisites. In: Lambkin, M., Foxall, G., Van Raaij, F., Heilbrunn, B. (eds.): European Perspectives on Consumer Behaviour, Prentice Hall, London (1998)

6. Eagly, A.H., Chaiken, S.: The Psychology of Attitudes. Forth Worth: Harcourt Brace Jovanovich (1993)

7. Efron, B., Tibshirani, R.J.: An Introduction to the Bootstrap. Chapman \& Hall. (1993)

8. Fernández Salido J.M., Murakami S.: Extending Yager's orness concept for the OWA aggregators to other mean operators. Fuzzy Sets and Systems, 139(3) (2003) 515-542

9. Filev D. and Yager R.R.: On the issue of obtaining OWA operator weights. Fuzzy Sets and Systems, 94 (1998) 157-169

10. Fishbein, M., Ajzen, I.: Belief, Attitude, Intention and Behavior: An Introduction to Theory and Research. Reading, MA: Addison-Wesley (1975)

11. Friedman, M.: Consumer Boycotts: Effecting Change Through the Marketplace and the Media. Routledge, London (1999)

12. Han, C.M.: The Role of Consumer Patriotism in the Choice of Domestic versus Foreign Products. Journal of Advertising Research, 28 (3) (1988) 25-32

13. Holbrook, M.B., Batra, R.: Assessing the Role of Emotions as Mediators of Consumer Responses to Advertising. Journal of Consumer Research, 14 (December) (1987) 404-420

14. Isen, A.M.: Some Ways in Which Affect Influences Cognitive Processes: Implications for Advertising and Consumer Behavior. In Cafferata, P., Tybout, A.M. (eds.): Cognitive and Affective Responses to Advertising, Lexington Books, Toronto (1989)

15. Klein, J.G., Ettenson, R., Morris, M.D.: The Animosity Model of Foreign Product Purchase: An Empirical Test in the People's Republic of China. Journal of Marketing, 62 (1998) 89-100

16. Liefeld, J.P.: Experiments on Country-of-Origin Effects: Review and Meta-Analysis of Effect Size. In: Papadopoulos, N., Heslop, L.A. (eds.): Product-Country Images: Importance and Role in International Marketing, International Business Press, New York (1993)

17. Obermiller, C., Spangenberg, E.: Exploring the Effects of Country of Origin Labels: An Information Processing Framework. Advances in Consumer Research, 16 (1989) 454-459 
18. Papadopoulos, N., Heslop, L.A.: Product-Country Images: Importance and Role in International Marketing, International Business Press, New York (1993)

19. Peter, J.P., Olson, J.C., Grunert, K.G.: Consumer Behaviour and marketing Strategy: European Edition, McGraw Hill, London (1999)

20. Peterson, R.A., Hoyer, W.D., Wilson, R.W.: The Role of Affect in Consumer Behavior: Emerging Theories and Applications. Lexington Books, Toronto (1986)

21. Shimp, T.A., Sharma, S.: Consumer Ethnocentrism: Construction and Validation of the CETSCALE. Journal of Marketing Research, 24 (August) (1987) 280-289

22. Stayman, D.M., Aaker, D.A.: Are All the Effects of Ad-Induced Feelings Mediated by Aad?. Journal of Consumer Research, 15 (December) (1988) 368-373

23. Torra V.: The weighted OWA operator. International Journal of Intelligent Systems, 12 (1997) 153-166

24. Torra V.: Learning weights for the quasi-weighted means. IEEE Transactions on Fuzzy Systems, 10(5) (2002) 653-666

25. Tulving, E., Thomson, D.M.: Encoding specificity and retrieval processes in episodic memory. Psychological Review, 80 (1973) 352-373

26. Verlegh, P.W.J.: Country-of-Origin Effects on Consumer Product Evaluations. Doctoral Dissertation, University of Wageningen (2001)

27. Verlegh, P.W.J., Steenkamp, J.-B.E.M.: A review and meta-analysis of country-of-origin research. Journal of Economic Psychology, 20 (5) (1999) 521-546

28. Watson, D., Clark, L.A., Tellegen, A.: Development and Validation of Brief Measures of positive and Negative Affect: The PANAS Scales. Journal of Personality and Social Psychology, 54 (6) (1988) 1063-1070

29. Yager, R.R.: An ordered weighted averaging operators in multicriteria decision making. IEEE Transactions on Systems, Man and Cybernetics, 18 (1988) 183-190

30. Yager, R.R. and Kacprzyck J.: The ordered weighted averaging operator, Theory and Applications, Kluwer Academic Publisher: Boston Dordecht London (1997) 